\title{
Farnesoid $X$ receptor and liver $X$ receptors regulate Oct $3 / 4$ expression by multiple feedback regulating system in normal renal-derived cells and renal adenocarcinoma cells
}

\author{
Tomofumi Fujino, Kouta Sugizaki, Rei Kato, Masaki Beppu, Satoshi Murakami, \\ Hyunjung Lee, Toshiyuki Oshima and Makio Hayakawa
}

\author{
Department of Hygiene and Health Sciences, School of Pharmacy, Tokyo University of Pharmacy and Life Sciences, \\ 1432-1 Horinouchi, Hachioji 192-0392, Japan
}

(Received August 29, 2019; Accepted November 15, 2019)

\begin{abstract}
In this study, we found that nuclear receptors FXR and LXR (originally characterized as regulatory factors involved in cholesterol/bile acid homeostasis) regulate the expression of Oct3/4, a marker for cell differentiation, in both normal renal-derived cell line HK-2 and renal adenocarcinoma cell line ACHN. Down-regulation of Oct3/4 expression by activating FXR and LXR occurs only in normal renal cell-derived HK-2 cells. We also found that the RNA-binding protein, ELAVL2, oppositely regulates Oct3/4 expressions in HK-2 and ACHN cells. Moreover, we revealed that LXR-alpha and LXR-beta regulate each other's expression. Although an LXR-beta-specific agonist is assumed to be the basis for an anti-arteriosclerotic drug that only stimulates reverse cholesterol transport, our findings show that the development of such an anti-arteriosclerotic drug would require further elucidation of the complex mechanism of LXR-alpha and LXR-beta regulation.
\end{abstract}

Key words: Farnesoid x receptor, Liver x receptor, Oct3/4, Cell differentiation

\section{INTRODUCTION}

Inhibition of cell proliferation is a common approach in cancer therapy. Given that stimulation of cell differentiation is associated with the inhibition of cell proliferation (Ju et al., 2015; Guo et al., 2009; Kremer-Tal et al., 2007; Andrews et al., 1994; Trieb et al., 1994), stimulation of cell differentiation of carcinoma cells is useful for cancer therapy. Our previous study showed that farnesoid X receptor (FXR), a bile acid-activated nuclear receptor (Makishima et al., 1999; Parks et al., 1999; Wang et al., 1999) which is involved in cholesterol/bile acid homeostasis (Zhang and Edwards, 2008), stimulates cell proliferation by down-regulating the expression of cyclin-dependent inhibitors p16/INK4a and p21/Cip1 in hepatocellular carcinoma cells and renal adenocarcinoma cells (Fujino et al., 2017, 2015, 2012). In contrast, FXR does not affect cell proliferation of normal hepatocyte- and renal cell-derived cell line (Fujino et al., 2017, 2015). FXR was originally characterized as a transcriptional factor that suppresses the expression of CYP7A1, a rate-limiting enzyme of the synthesis of bile acid (Zhang and Edwards, 2008), and liver X receptor (LXR) alpha, an oxysterol-activated nuclear receptor (Janowski et al., 1996), stimulates the expression of CYP7A1 (Chiang et al., 2001). Since our previous study shows that FXR stimulates the expression of hepatocyte nuclear factor-4alpha (Fujino et al., 2012), known to be involved in cell differentiation (Li et al., 2000), we examined whether FXR and LXR regulates cell differentiation in the present study.

It is well known that Oct3/4 is a cell differentiation marker (Okamoto et al., 1990; Rosner et al., 1990; Schöler et al., 1990). Oct3/4 belongs to the POU family of transcription factors and regulates the expression of pluripotency-associated genes in cooperation with Sox2 (Nichols et al., 1998). Conditional repression of gene encoding Oct $3 / 4$ in ES cells leads to their differentiation (Niwa et al., 2000). Suppression of Oct3/4 expression reduces cell proliferation of mouse embryonic stem cells (Kanai et al., 2015). Oct3/4 is also involved in cancer cell growth. For example, Oct3/4 knockdown suppresses the proliferation of hepatocellular carcinoma cells (Tomizawa

Correspondence: Tomofumi Fujino (E-mail: tfujino@ps.toyaku.ac.jp) 
et al., 2018).

In addition to the regulations at transcriptional levels, posttranscriptional regulation by RNA-binding proteins has been highlighted in cell growth and differentiation in mammalian tissues. There are increasing lines of evidence that suggest that RNA dysregulation is criti$\mathrm{cal}$ in the pathogenesis of several diseases including cancer (Yano et al., 2016). ELAVL2, one of the members of ELAVL family that acts as the RNA-binding proteins is known to orchestrate the post-transcriptional regulation of gene expression. Importantly, ELAVL2 is known to up-regulate $\mathrm{p} 21 / \mathrm{Cip} 1$ expression in esophageal squamous cell carcinoma (Zhao et al., 2019).

We found that the nuclear receptors FXR and LXR regulate the expression of Oct $3 / 4$ in normal renal cell-derived cell line HK-2 and renal adenocarcinoma cell line ACHN. Down-regulation of Oct3/4 expression by activation of FXR or LXR occurred only in normal renal cellderived HK-2 cells. Down-regulation of Oct3/4 in renal adenocarcinoma ACHN cells by an inverse agonist for LXR is a transient event arising from loop regulation between Oct3/4 and its upstream factor ELAVL2. Moreover, we revealed that LXR-alpha and LXR-beta regulate each other's expression.

\section{MATERIALS AND METHODS}

\section{Materials}

Antibodies (Abs) specific for $\beta$-actin, LXR (H-144, sc-13068), and Oct3/4 (C-10, sc-5279) were purchased from Santa Cruz Biotechnology (Dallas, TX, USA). An antibody specific for ELAVL2 (ab171414) was purchased from Abcam (Cambridge, UK). ECL ${ }^{\mathrm{TM}}$ anti-mouse $\mathrm{IgG}$, horseradish peroxidase-linked whole antibody (from sheep) and ECL ${ }^{\mathrm{TM}}$ anti-rabbit IgG, horseradish peroxidase-linked whole antibody (from donkey) were purchased from GE Healthcare (Buckinghamshire, UK). Oct3/4, ELAVL2, LXR-alpha, and LXR-beta siRNA were purchased from Qiagen (Hulsterweg, NLD). GW4064 was purchased from TOCRIS (Bristol, UK). GW3965 and SR9243 were purchased from Sigma Aldrich (St. Louis, MO, USA). The human renal adenocarcinoma cell line ACHN and human normal renal-derived cell line HK-2 were obtained from ATCC.

\section{Cell culture}

HK-2 is a proximal tubular cell line derived from normal human kidney. The cells were immortalized by transduction with human papilloma virus 16 (Ryan et al., 1994) (ATCC No.CRL-2190). ACHN is a renal adenocarcinoma cell line derived from a 22-year-old man (ATCC
No. CRL-1611). These cell lines were maintained in Dulbecco's modified eagle medium (DMEM) containing $10 \%$ fetal calf serum (FCS), 50 units $/ \mathrm{mL}$ penicillin $\mathrm{G}$ sodium salt, and $50 \mu \mathrm{g} / \mathrm{mL}$ streptomycin sulfate and cultured in a humidified atmosphere of $8.5 \% \mathrm{CO}_{2}$ at $37^{\circ} \mathrm{C}$.

\section{RNA interference experiments}

In order to knockdown endogenous LXR-alpha, LXRbeta, ELAVL2, and Oct $3 / 4$ cells were seeded on $60-\mathrm{mm}$ dishes at a density of $5.0 \times 10^{5} \mathrm{HK}-2$ cells or $3.0 \times 10^{5}$ ACHN cells per dish and transfected with siRNA against LXR-alpha, LXR-beta, ELAVL2, and Oct3/4 (50 nM) using HiPerfect Transfection Reagent (Qiagen) according to the manufacturer's instructions. After incubation for $24 \mathrm{hr}$, cell extracts for immunoblotting analyses or total RNAs for real-time PCR analyses were prepared. In the RNA interference experiments, "Nonsilencing Control" siRNA (\#1022076) from Qiagen was used as a control.

\section{Quantification of mRNAs}

Quantification of mRNA was performed using realtime PCR. Briefly, $4 \mu \mathrm{g}$ of total RNA was reverse-transcribed using ReverTra Ace qPCR RT Master Mix (Toyobo, Osaka, Japan). The resultant cDNA was subjected to real-time PCR analysis using a TaqMan Gene Expression Assay kit (Applied Biosystems, Tokyo, Japan). mRNA levels were determined using TaqMan assay mixtures for ELAVL2 (Hs00270011), Oct3/4 (Hs04260367), LXR-alpha (Hs00172885), LXR-beta (Hs00173195), and $\beta$-actin (4310881E). Amplification and quantification were performed using the StepOne Real-Time PCR System (Applied Biosystems). ELAVL2, Oct3/4, LXR-alpha, and LXR-beta mRNA levels were normalized to those of $\beta$-actin as an internal control.

\section{Construction of expression vectors containing open reading frames of ELAVL2, LXR-alpha, and LXR-beta}

The expression vectors containing open reading frames (ORF) of ELAVL2 (NM_004432.5), LXR-alpha (NM_005693.4), and LXR-beta (NM_007121.7) were constructed as follows. cDNAs encoding full-length human ELAVL2, LXR-alpha, or LXR-beta were synthesized by GINEWIZ (Saitama, Japan) and subcloned into pcDNA3.1(+). The resultant plasmids were termed pcELAVL2, pc-LXR-alpha, and pc-LXR-beta.

\section{Ectopic expression of ELAVL2, LXR-alpha, and LXR-beta}

HK-2 cells were seeded at $5.0 \times 10^{5}$ cells or ACHN cells were seeded at $3.0 \times 10^{5}$ cells $/ 60-\mathrm{mm}$ dish and cul- 
Regulation of Oct3/4 expression by FXR and LXR

tured for $24 \mathrm{hr}$. Cells were then transfected with pcDNA3.1(+), pc-ELAVL2, pc-LXR-alpha or pc-LXR-beta by using Lipofectamine 2000 transfection reagent (Invitrogen, Carlsbad, CA, USA).

\section{Immunoblotting}

Cells were washed with PBS, and cell extracts were prepared using SDS sample buffer without loading dye. After normalization of protein content via the protein assay, the dye was added to samples. The samples were subjected to SDS-PAGE and immunoblotting analyses. For detection of Oct3/4, ELAVL2, LXR-alpha, LXR-beta, and $\beta$-actin, PVDF membranes were incubated with primary antibody $(1: 100)$ for $2 \mathrm{hr}$. Immunocomplexes on the PVDF membranes were visualized using enhanced chemiluminescence western blotting detection reagents (GE Healthcare). Quantification of bands was conducted using densitometric analysis (IMAGE GAUGE 4.0).

\section{Statistical analysis}

Data were analyzed using Student's t-test and are presented as the mean \pm S.E.M. of three experiments performed in triplicate.

\section{RESULTS}

\section{FXR down-regulates Oct3/4 expression in normal kidney-derived cell line HK-2}

The proximal tubular cell line derived from normal human kidney, HK-2, and renal adenocarcinoma cell line, ACHN, were treated with GW4064, synthetic FXR agonist (Maloney et al., 2000), and then the Oct3/4 level was determined. As shown in Fig. 1A and B, GW4064 decreased Oct $3 / 4$ level in HK-2 cells, but did not alter the Oct3/4 expression in ACHN cells. These results suggest that FXR stimulates cell differentiation of normal renal cells but does not affect differentiation of renal adenocarcinoma cells.

\section{Both the LXR agonist and inverse agonist down-regulate Oct3/4 expression in HK-2 cells while only the LXR inverse agonist down- regulates Oct $3 / 4$ expression in ACHN cells}

After observing that FXR down-regulated Oct3/4 expression in normal renal cell-derived cell line HK-2, we examined whether LXR activation affects Oct3/4 expression. Similar to FXR ligand-treatment, LXR agonist GW3965 (Collins et al., 2002) lowered Oct3/4 level in HK-2 cells (Fig. 1C-a), but did not affect the Oct3/4 level of ACHN cells (Fig. 1C-b). Unexpectedly, LXR inverse agonist SR9243 (Flaveny et al., 2015) down-reg- ulated Oct3/4 expression in both HK-2 and ACHN cells (Fig. 1C, 1D-a, and "Day 3" of 1D-b). In renal adenocarcinoma ACHN cells, the inverse agonist-induced decrease in Oct3/4 level was restored after longer incubation ("Day 7" of Fig. 1D-b), indicating that down-regulation of Oct $3 / 4$ expression is a transient event.

\section{ELAVL2 and Oct3/4 regulate each other's expressions}

As shown in Fig. 1, the LXR inverse agonist-induced decrease in Oct3/4 level was restored after longer incubation, indicating that down-regulation of Oct3/4 expression is temporary in renal adenocarcinoma ACHN cells. This is assumed to be the mechanism for maintaining an undifferentiated state of renal adenocarcinoma cells. Previously, we obtained the preliminary data suggesting that ELAVL2, a neural-specific RNA-binding protein, regulates the Oct $3 / 4$ expression (our unpublished data). Since ELAVL2 is known to up-regulate p21/Cip1 expression, we hypothesized that ELAVL2 may be involved in the regulation of Oct $3 / 4$ expression.

The ELAVL2 and Oct3/4 knockdown assays in HK-2 cells (Fig. 2A) show that knockdown of one reduces expression of the other, suggesting that positive feedback regulations exist between ELAVL2 and Oct3/4 in HK-2 cells. Conversely, while Oct $3 / 4$ knockdown in ACHN cells reduces ELAVL2 expression, ELAVL2 knockdown increases Oct3/4 expression (Fig. 2A). These results indicate that ELAVL2 expression in ACHN cells is dependent on Oct3/4 as that in HK-2 cells while ELAVL2 exerts suppressive effect on Oct3/4 expression. However, when ELAVL2 was overexpressed (Fig. 2B), Oct3/4 expression was suppressed not only in ACHN cells but also in HK-2 cells (Fig. 2C). We can postulate that ELAVL2 may target the RNA molecule that leads to the elevation of Oct $3 / 4$ mRNA levels in HK-2 cells. On the other hand, in ACHN cells, ELAVL2 may target the different RNA molecule, resulting in the down-regulation of Oct3/4 mRNA. When the ELAVL2 expression is up-regulated, this suppressive pathway on Oct3/4 mRNA may also be activated in HK-2 cells.

\section{Knockdown of LXR-alpha does not affect Oct3/4 expression of HK-2 cells but slightly increases that of ACHN cells, while knockdown of LXR- beta decreases Oct $3 / 4$ expression in HK-2 and ACHN cells}

Given that Oct3/4 expression in HK-2 cells was downregulated when LXR was either activated or repressed and that Oct3/4 expression in ACHN cells was downregulated by the LXR inverse agonist, we next examined 

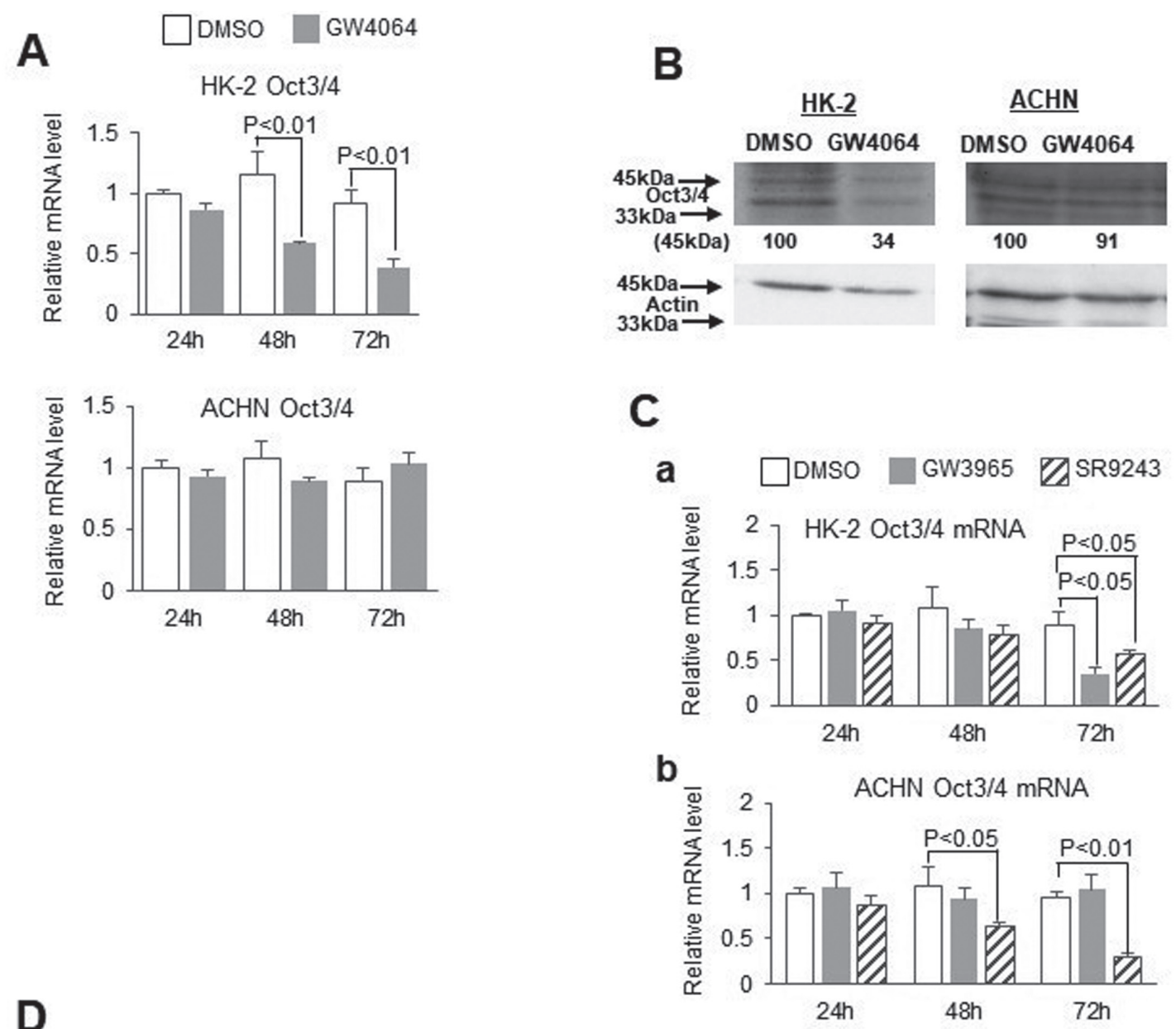

a

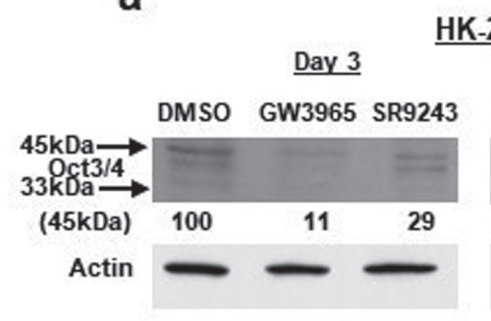

HK-2

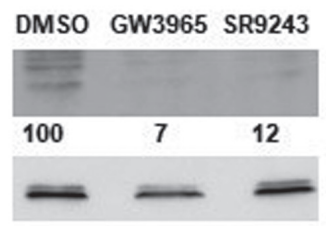

b

Fig. 1. Alteration of Oct $3 / 4$ expression in normal renal cell-derived HK-2 cells and renal adenocarcinoma ACHN cells treated with FXR agonist, LXR agonist, or LXR inverse agonist. A and B: HK-2 cells seeded at $5.0 \times 10^{5}$ cells or ACHN cells seeded at $3.0 \times 10^{5}$ cells/ 60 -mm dish were treated with $0.1 \%$ of DMSO or $0.1 \%$ of DMSO containing $10 \mu \mathrm{M} \mathrm{GW} 4064$. After 24,48 , and $72 \mathrm{hr}$, total RNA was extracted, and quantification of Oct3/4 mRNA was performed as described in Materials and Methods. Data are presented as the mean \pm S.E.M. of three experiments performed in triplicate and analyzed using Student's t-test (A). After $72 \mathrm{hr}$, cell extracts were subjected to immunoblotting to detect Oct3/4 and $\beta$-actin protein (B). C: HK-2 cells seeded at $5.0 \times 10^{5}$ cells or ACHN cells seeded at $3.0 \times 10^{5}$ cells $/ 60-\mathrm{mm}$ dish were treated with $0.1 \%$ of DMSO, or $0.1 \%$ of DMSO containing $5 \mu \mathrm{M}$ GW3965 or SR9243. After 24,48 , and $72 \mathrm{hr}$, total RNA was extracted, and quantification of Oct3/4 mRNA was performed as described in Materials and Methods. Data are presented as the mean \pm S.E.M. of three experiments performed in triplicate and analyzed using Student's t-test. D: HK-2 cells seeded at $1.0 \times 10^{5}$ cells or ACHN cells seeded at $6.0 \times 10^{4}$ cells/ 60 -mm dish were treated with $0.1 \%$ of DMSO, or $0.1 \%$ of DMSO containing $5 \mu \mathrm{M} \mathrm{GW3965}$ or SR9243. After 3 and 7 days, cell extracts were subjected to immunoblotting to detect Oct $3 / 4$ and $\beta$-actin protein. 
A HK-2
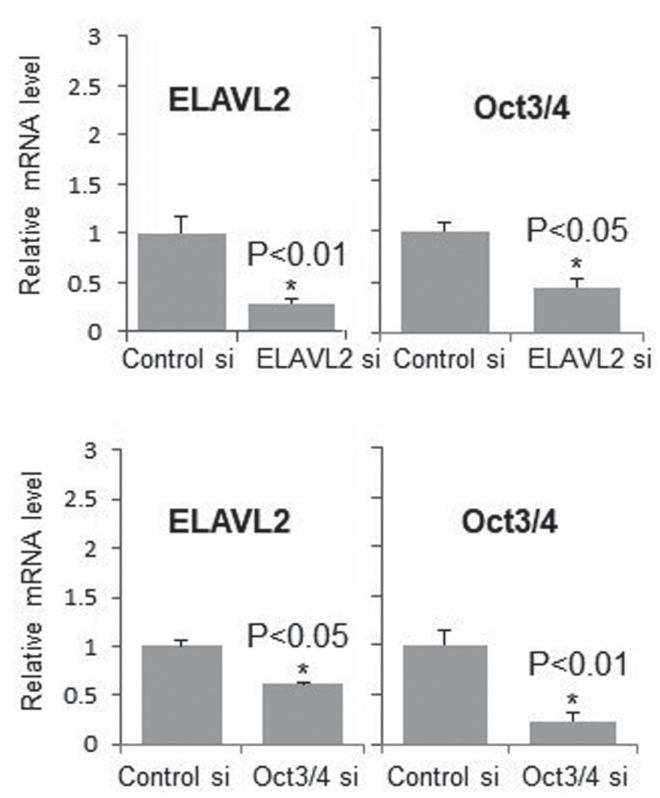

B

\section{HK-2}

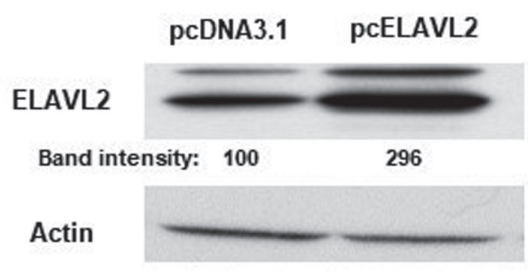

\section{ACHN}

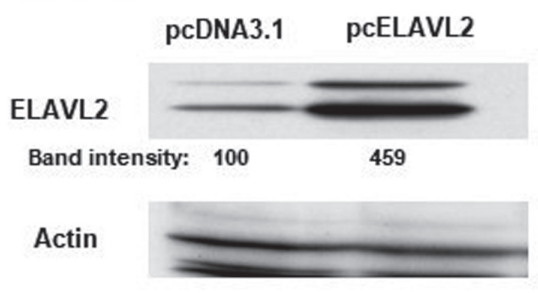

\section{ACHN}
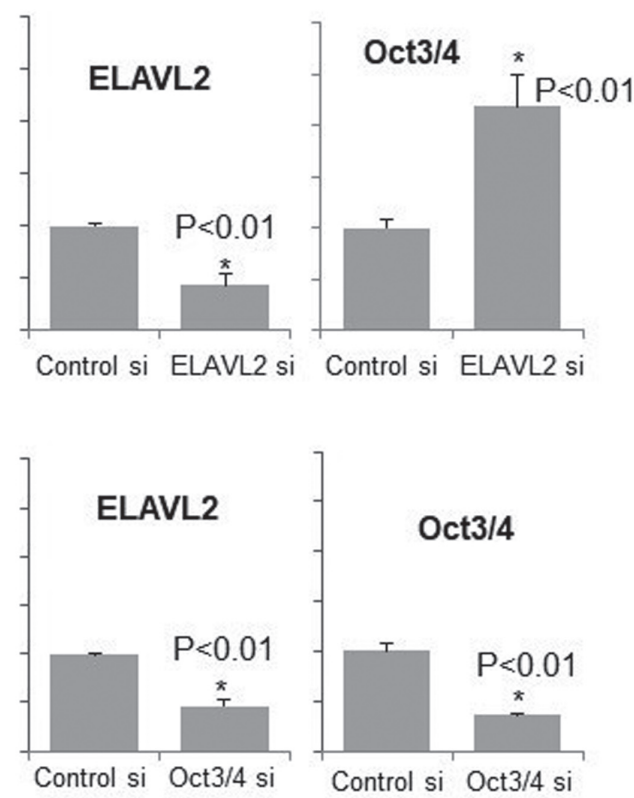

C
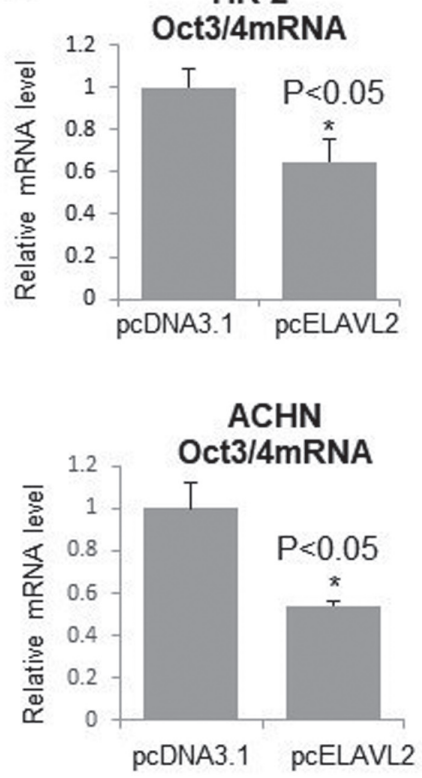

Fig. 2. Alteration of Oct $3 / 4$ expression by knockdown or ectopic expression of ELAVL2. A: HK-2 cells seeded at $5.0 \times 10^{5}$ cells or ACHN cells seeded at $3.0 \times 10^{5}$ cells/60-mm dish were transfected with control or ELAVL2 siRNA. After $24 \mathrm{hr}$, total RNA was extracted, and quantification of ELAVL2 and Oct3/4 was performed as described in Materials and Methods. Data were analyzed using Student's t-test and are presented as the mean \pm S.E.M. of three experiments performed in triplicate. $\mathrm{B}$ and $\mathrm{C}$ : HK-2 cells seeded at $5.0 \times 10^{5}$ cells or ACHN cells seeded at $3.0 \times 10^{5}$ cells $/ 60$-mm dish were transfected with pcDNA3.1(+) or pc-ELAVL2 for $24 \mathrm{hr}$. Cell extracts from HK-2 and ACHN cells were subjected to immunoblotting to detect ELAVL2 and $\beta$-actin protein (B). total RNA was extracted, and quantification of Oct3/4 was performed as described in Materials and Methods (C). Data were analyzed using Student's t-test and are presented as the mean \pm S.E.M. of three experiments performed in triplicate. 
A

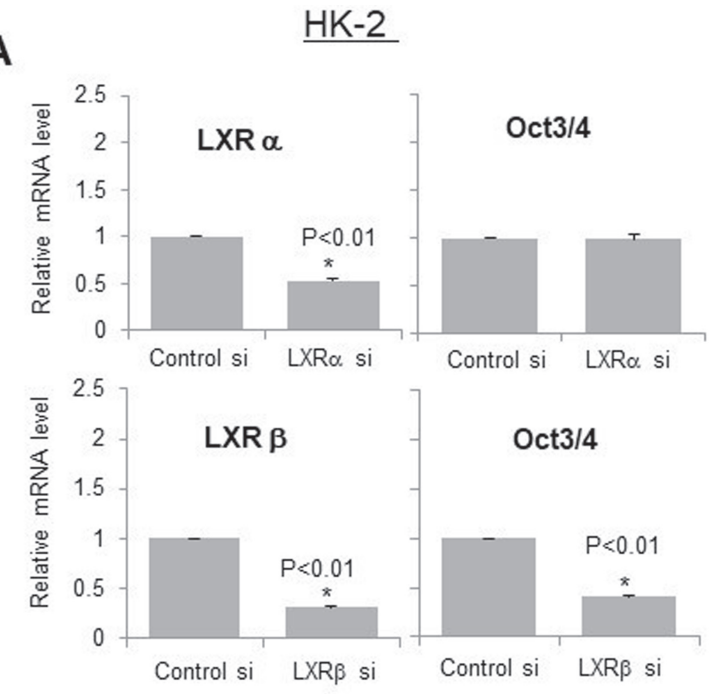

B
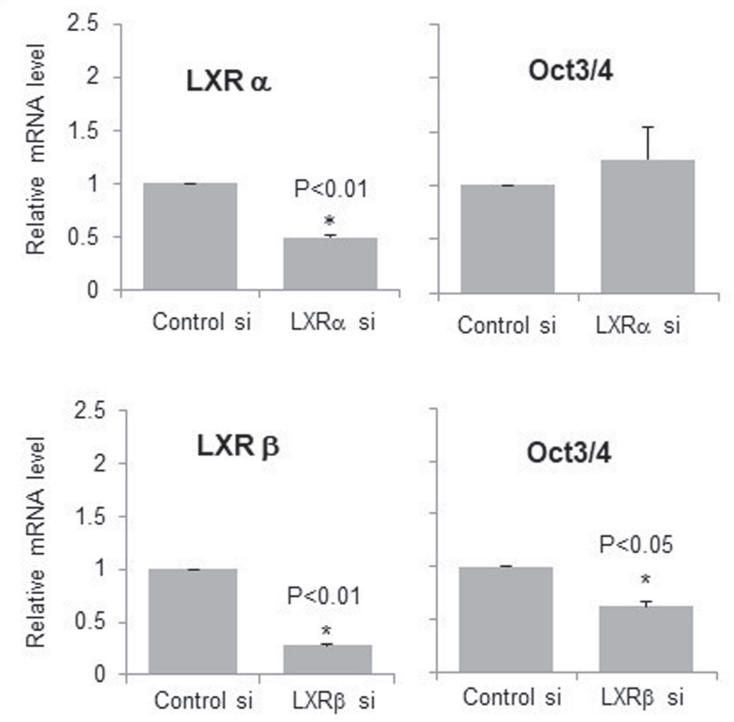

Fig. 3. Alteration of Oct $3 / 4$ expression in HK-2 and ACHN cells transfected with LXR-alpha and LXR-beta siRNA. HK-2 cells seeded at $5.0 \times 10^{5}$ cells $(\mathrm{A})$ or $\mathrm{ACHN}$ cells seeded at $3.0 \times 10^{5}$ cells $(\mathrm{B}) / 60-\mathrm{mm}$ dish were transfected with control, LXR-alpha,or LXR-beta siRNA. After $24 \mathrm{hr}$, total RNA was extracted, and quantification of Oct3/4 was performed as described in Materials and Methods. Data were analyzed using Student's t-test and are presented as the mean \pm S.E.M. of three experiments performed in triplicate.

how LXR-alpha and LXR-beta (two endogenous isofor$\mathrm{ms}$ of LXR) are each involved in the regulation of Oct $3 / 4$ expression, and how agonists affect their activity.
HK-2 and ACHN cells were transfected with siRNA against LXR-alpha and LXR-beta in separate experiments. In HK-2 cells, LXR-beta knockdown decreased Oct3/4 level, while LXR-alpha knockdown did not affect the Oct3/4 expression (Fig. 3A). In ACHN cells, LXR-beta knockdown lowered the Oct3/4 level similar to HK-2 cells, while LXR-alpha knockdown stimulated, Oct3/4 expression slightly, albeit not significantly (Fig. 3B). The results from the LXR knockdown assays indicate that LXR-beta stimulates Oct3/4 expression in both normal renal-derived HK-2 cells and renal adenocarcinoma ACHN cells, whereas LXR-alpha does not affect the Oct3/4 expression of HK-2 cells but slightly downregulates that of $\mathrm{ACHN}$ cells.

\section{Ectopic expression of LXR-alpha with exogenous LXR agonist down-regulates LXR- beta expression and ectopic expression of LXR- beta up-regulates LXR-alpha expression in HK-2 cells}

To examine the agonist-dependency of the LXR regulation of Oct3/4 expression in HK-2 cells, ectopic expression of LXR-alpha and -beta was performed. As shown in Fig. 4C-a, ectopic expression of LXR-alpha for $24 \mathrm{hr}$ with LXR agonist GW3965 (Fig. 4A) did not affect Oct3/4 expression. Interestingly, the LXR-beta level was lowered by ectopic expression of LXR-alpha for $24 \mathrm{hr}$ with exogenous LXR agonist (Fig. 4C-c). The down-regulation of LXR-beta expression by LXR-alpha is agonist-dependent, since ectopic expression of LXR-alpha without exogenous agonist (Fig. 4A) did not lower the LXR-beta level (Fig. 4C-c). On the other hand, ectopic expression of LXR-beta without exogenous agonist (Fig. 4A) stimulated Oct3/4 expression (Fig. 4C-b), consistent with the knockdown of endogenous LXR-beta decreasing the Oct3/4 level of HK-2 cells (Fig. 3A). The ectopic expression of LXR-beta without exogenous agonist (Fig. 4A) also stimulated LXR-alpha expression (Fig. 4C-d). Unexpectedly, addition of exogenous agonist repressed the increase in Oct3/4 (Fig. 4C-b) and LXR-alpha (Fig. $4 \mathrm{C}-\mathrm{d}$ ), indicating that LXR-beta activity in $\mathrm{HK}-2$ cells was inhibited by exogenous agonist.

Treatment of HK-2 cells with exogenous LXR agonist has several effects as follows: LXR-alpha is activated, resulting in down-regulated LXR-beta expression (Oct3/4 decreases); LXR agonist represses LXR-beta activity (Oct3/4 decreases); and down-regulated LXRalpha expression restores the decreased LXR-beta level (Oct3/4 increases). Thus, the net down-regulation of Oct3/4 expression by exogenous LXR agonist occurs as the combination of these changes to Oct $3 / 4$ expression, 

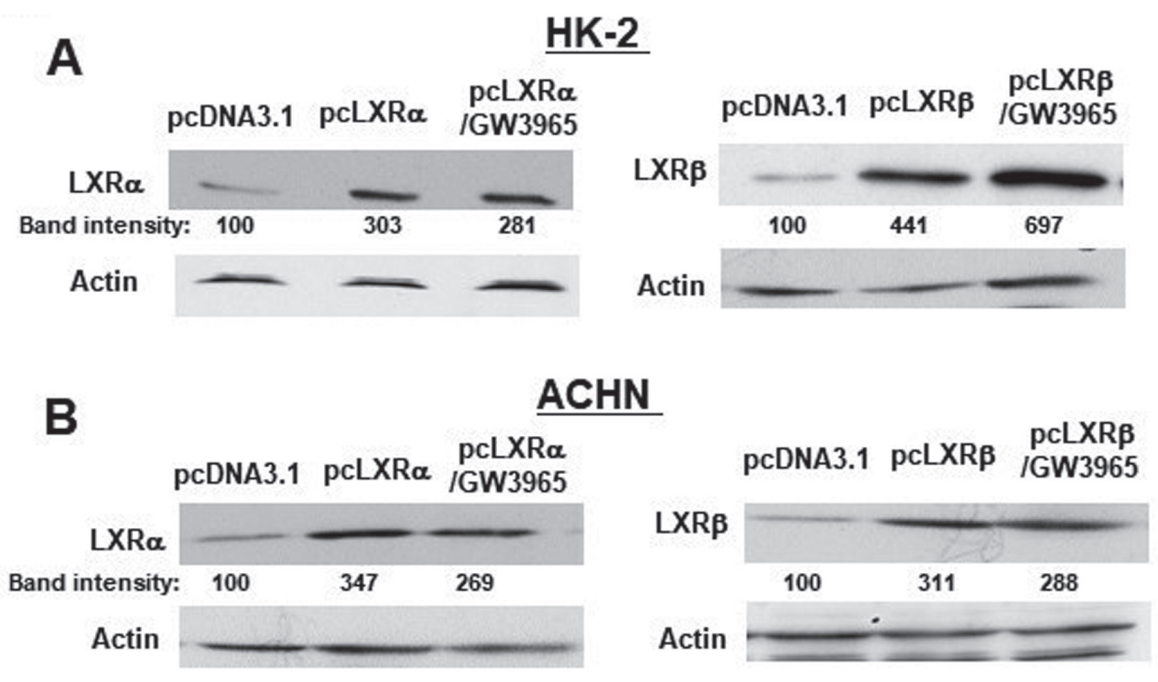

C

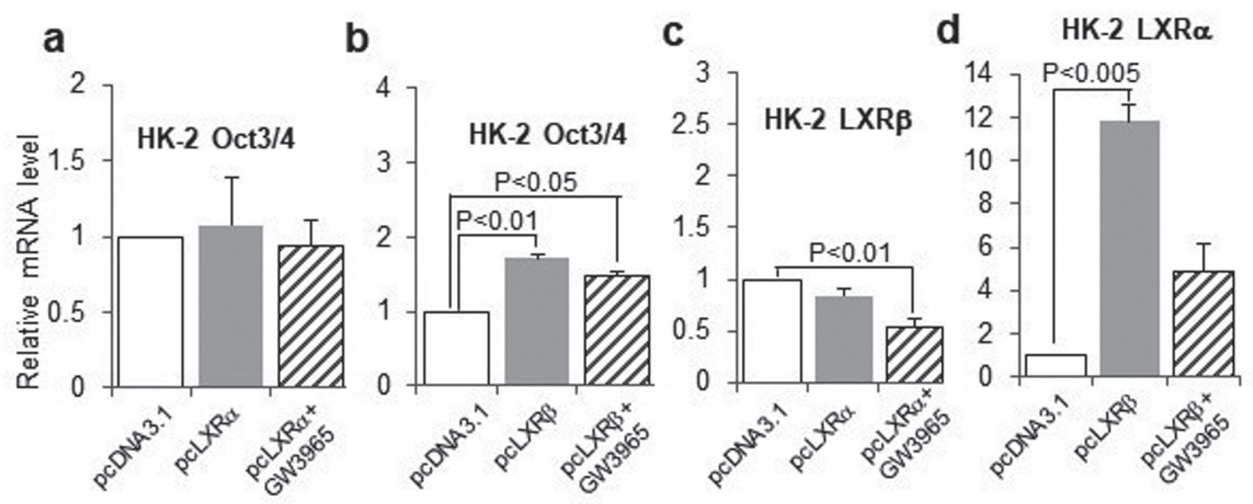

D
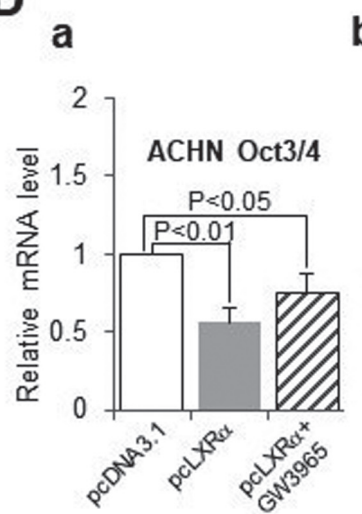

b

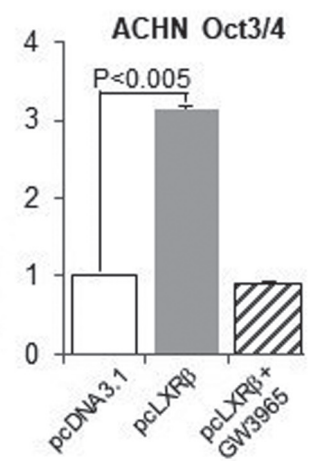

C

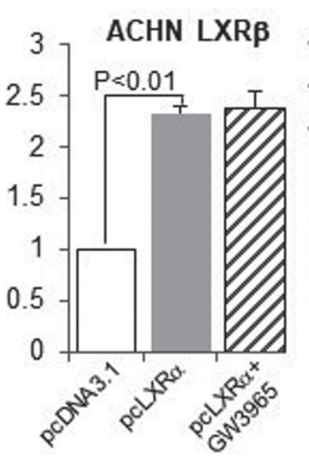

d

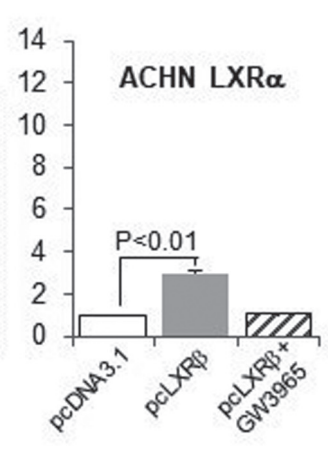

Fig. 4. Alteration of Oct3/4, LXR-alpha, and LXR-beta expression by ectopic expression of LXR-alpha and LXR-beta in HK-2 and ACHN cells. HK-2 cells seeded at $5.0 \times 10^{5}$ cells or ACHN cells seeded at $3.0 \times 10^{5}$ cells $/ 60$-mm dish were transfected with pcDNA3.1(+), pc-LXR-alpha, or pc-LXR-beta in the presence or absence of $5 \mu \mathrm{M} \mathrm{GW} 3965$ for 24 hr. The cell extracts from HK-2 cells (A) and ACHN cells (B) were subjected to immunoblotting to detect LXR-alpha, LXR-beta, and $\beta$-actin protein. The total RNA from HK-2 cells (C) and ACHN cells (D) was extracted, and quantification of Oct3/4, LXR-alpha and LXR-beta was performed as described in Materials and Methods. Data were analyzed using Student's t-test and are presented as the mean \pm S.E.M. of three experiments performed in triplicate. 
A

HK-2 cells with LXR agonist

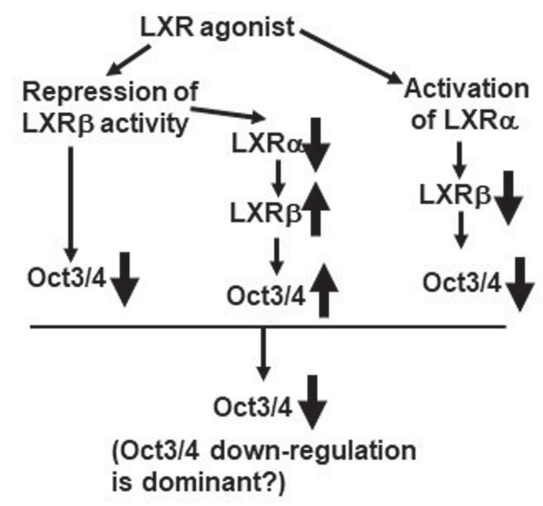

C

ACHN cells with $L X R$ agonist

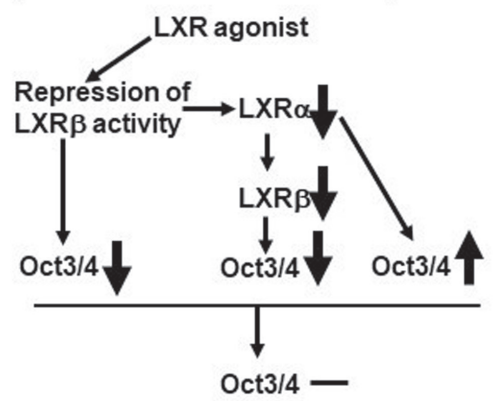

(Oct3/4 down-regulation and up-regulation counteract ?)
B

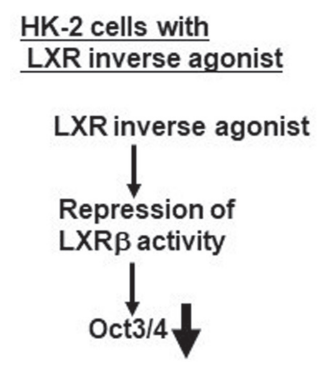

D

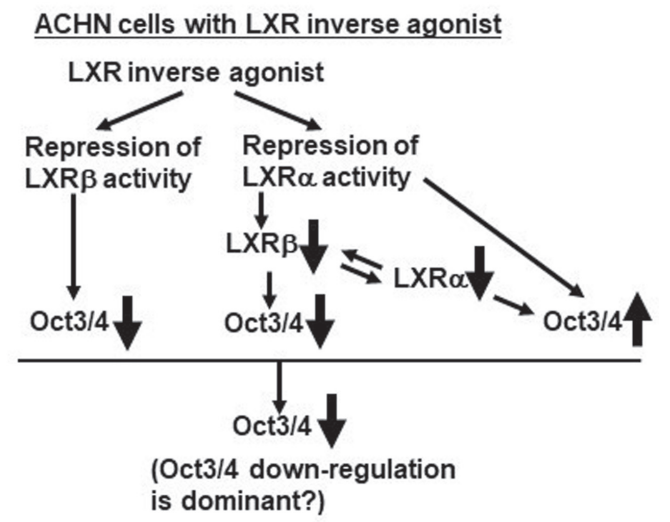

Fig. 5. Scheme of alteration of LXR-alpha, LXR-beta, and Oct $3 / 4$ expression in HK-2 and ACHN cells treated with LXR agonist and LXR inverse agonist.

although down-regulation of Oct $3 / 4$ is probably dominant to up-regulation of Oct3/4 (Fig. 5A). When treated with inverse agonist for LXRs, endogenous LXR-beta activity in HK-2 cells is inhibited, resulting in an Oct3/4 decrease (Fig. 5B).

\section{Ectopic expression of LXR-alpha without exogenous LXR agonist stimulates LXR-beta expression and ectopic expression of LXR-beta up-regulates LXR-alpha expression in ACHN cells}

To determine why Oct $3 / 4$ expression was not altered by the LXR agonist in ACHN cells, ectopic expression of LXR-alpha and -beta was performed in ACHN cells. As shown in Fig. 4B and D, ectopic expression of LXR-alpha for $24 \mathrm{hr}$ without exogenous LXR agonist (Fig. 4B) decreased the Oct3/4 level (Fig. 4D-a). In the cells, the LXR-beta mRNA level increased (Fig. 4D-c). Exogenous LXR agonist did not alter this Oct3/4 decrease and LXRbeta increase (Fig. 4D-a and Fig. 4D-c), suggesting that LXR-alpha is fully activated by endogenous agonist in ACHN cells, which is different from HK-2 cells and consistent with the observation that endogenous LXR-alpha knockdown slightly increased the Oct $3 / 4$ level of ACHN cells (Fig. 3B). Similar to HK-2 cells, ectopic expression of LXR-beta stimulated Oct3/4 expression without exogenous agonist (Fig. 4D-b), which is consistent with the endogenous LXR-beta knockdown decreasing the Oct3/4 
level of ACHN cells (Fig. 3B). Moreover, the ectopic expression of LXR-beta without exogenous agonist (Fig. 4B) stimulated LXR-alpha expression (Fig. 4D-d) and the addition of exogenous agonist repressed the increase in Oct3/4 and LXR-alpha (Fig. 4D-b and Fig. 4D-d), indicating that LXR-beta activity in ACHN cells was inhibited by exogenous agonist, as in HK-2 cells.

Treatment of ACHN cells with exogenous LXR agonist has several effects as follows: LXR agonist represses LXR-beta activity, resulting in a decrease in the Oct3/4 and LXR-alpha levels. The down-regulation of LXR-alpha restores decreased Oct3/4 level and reduces the LXR-beta level, resulting in down-regulation of Oct $3 / 4$ expression. Thus, exogenous LXR agonist causes several adverse alterations of Oct $3 / 4$ level in ACHN cells. Consequently, exogenous LXR agonist marginally affects Oct $3 / 4$ expression by counteracting adverse alterations (Fig. 5C).

In contrast, the inverse agonist has the following effects: the inverse agonist represses LXR-alpha activity, increasing Oct3/4. Since LXR-alpha stimulates LXR-beta expression without exogenous agonist in ACHN cells, which is different from HK-2 cells, the inverse agonist also represses the LXR-beta expression that is induced by LXR-alpha, decreasing Oct3/4. Moreover, similar to HK-2 cells, the inverse agonist represses LXR-beta activity, decreasing the Oct3/4 and LXR-alpha levels. The down-regulation of LXR-alpha restores the decreased Oct3/4 level and reduces the LXR-beta level, resulting in down-regulation of Oct $3 / 4$ expression. Thus, the inverse agonist causes a number of adverse changes in Oct $3 / 4$ expression, although down-regulation of Oct $3 / 4$ is probably dominant to up-regulation of Oct3/4. Consequently, the inverse agonist for LXRs down-regulates Oct3/4 expression in ACHN cells (Fig. 5D).

\section{DISCUSSION}

In this study, we found that the nuclear receptors FXR and LXR-originally characterized as transcriptional factors involved in cholesterol/bile acid homeostasis (Zhang and Edwards, 2008; Chiang et al., 2001) - regulate the expression of Oct3/4, a marker for cell differentiation (Okamoto et al., 1990; Rosner et al., 1990; Schöler et al., 1990), in normal renal cell-derived cell line HK-2 and renal adenocarcinoma cell line ACHN. Down-regulation of Oct3/4 expression by activation of FXR or LXR occurred only in normal renal cell-derived HK-2 cells. The down-regulation of Oct3/4 in renal adenocarcinoma ACHN cells by an inverse agonist for LXR is a transient event arising from loop regulation between Oct3/4 and its upstream factor ELAVL2.

Besides the transcriptional control, we also focus on the posttranscriptional control of Oct3/4 expression by the RNA-binding protein, ELAVL2. As shown in Fig. 2, Oct3/4 expression is negatively regulated by ELAVL2 in ACHN cells whereas it is positively regulated by ELAVL2 in HK-2 cells. At present, we cannot fully explain why Oct3/4 expression is oppositely regulated by ELAVL2 in HK-2 and ACHN cells. It should be noted that ELAVL2 expression level in ACHN cells is approximately 10 -fold lower than HK-2 cells, while Oct3/4 level in ACHN cells is approximately 3 -fold higher than HK-2 cells (our unpublished data). We can assume that ELAVL2 may act on the different target RNAs in cells expressing Oct3/4 at lower levels, compared with those in cells expressing Oct $3 / 4$ at higher levels. In HK-2 cells, in which Oct3/4 is expressed at relatively lower level, ELAVL2 targets the RNA that upregulates Oct3/4. On the other hand, in ACHN cells, in which Oct3/4 is expressed at relatively higher level, ELAVL2 targets the RNA that downregulates Oct3/4. When overexpressed, ELAVL2 may dominantly target the RNA that downregulates Oct3/4.

The present study was performed using renal cell lines. Although it has been reported that Oct $3 / 4$ knockdown suppressed the proliferation of hepatocellular carcinoma cells in the condition of 3-day incubation (Tomizawa et al., 2018), whether suppressed proliferation restore by longer incubation is remained to be unknown. In fact, in the present study, 3-day incubation of ACHN cells with LXR inverse agonist caused a decrease in cell number, however, the cell number was restored after 4 days (data not shown). The present study also needs to be designed using hepatic cells.

Our previous study showed that FXR stimulates cell proliferation of hepatocellular carcinoma cells and renal adenocarcinoma cells by down-regulating the expressions of cyclin-dependent kinase inhibitor, p16/INK4a and p21/ Cip1, without affecting that of normal hepatocyte- and renal-derived cells (Fujino et al., 2012, 2015, 2017). Given that FXR down-regulates Oct3/4 expression in the normal renal-derived cell line HK-2 without affecting that in renal adenocarcinoma cell line ACHN (Fig. 1A and B), the significance of FXR transfers from the stimulation of cell differentiation in normal hepatocyte and renal cells to the stimulation of cell proliferation in hepatocellular carcinoma and renal adenocarcinoma cells. This transfer probably enhances carcinoma cell viability by repressing cell differentiation which inhibits cell proliferation and directly stimulating cell proliferation.

It was surprising that LXR agonist GW3965 inhibited 
LXR-beta-induced stimulation of Oct3/4 and LXR-alpha expression (Fig. 4C-b, -d and Fig. 4D-b, -d). We assume that LXR-beta is fully activated by an endogenous agonist specific for LXR-beta, and so the exogenous agonist competes with the endogenous agonist for the ligand binding domain. It is also interesting that LXR-alpha is active without exogenous agonist in renal adenocarcinoma ACHN cells (Fig. 4D-a, -c). It is known that renal cell carcinoma tissue contains higher levels of total cholesterol and cholesterol esters compared with normal kidney tissues (Gebhard et al., 1987). Thus, in renal adenocarcinoma cells, there probably exists a carcinoma-specific agonist of cholesterol derivatives which activates either LXR-alpha or LXR-alpha and LXR-beta. In contrast to LXR-beta activity, which is inhibited by exogenous agonist (Fig. 4D-b, -d), LXR-alpha activity is not affected by exogenous agonist (Fig. 4D-a, -c), suggesting that a carcinoma-specific agonist that activates LXR-alpha does not compete with exogenous agonist for the ligand binding domain. Moreover, ectopic expression of LXR-alpha induced an adverse change in ACHN cells compared to exogenous agonist-treated HK-2 cells (Fig. 4C-c and Fig. 4D-c), supporting the possibility of a carcinoma-specific agonist.

LXR is known to activate ATP-binding cassette transporters G5 (ABCG5) and G8 (ABCG8) in the intestine, which stimulates excretion of cholesterol (Repa et al., 2002), and LXR is also known to activate ABCA1 and ABCG1 expression in peripheral blood vessels, which stimulates reverse cholesterol transport (Venkateswaran et al., 2000; Kennedy et al., 2001). Although LXRs have such an anti-arteriosclerotic effect, LXR-alpha also stimulates the synthesis of fatty acid (Pawar et al., 2003), resulting in the development of arteriosclerosis. Thus, at present, an LXR-beta-specific agonist is assumed to be the basis for an anti-arteriosclerotic drug without side-effects (Koura et al., 2015). However, given that our findings show that LXR-alpha and LXR-beta regulate each other's expression, the development of such an anti-arteriosclerotic drug requires further elucidation of the complex mechanism of LXR-alpha and LXR-beta regulation.

\section{ACKNOWLEDGMENTS}

We thank Ken Ando for his helpful advice and discussion. This work was supported in part by a grant from the Japan Private School Promotion Foundation.

Conflict of interest---- The authors declare that there is no conflict of interest.

\section{REFERENCES}

Andrews, P.W., Damjanov, I., Berends, J., Kumpf, S., Zappavigna, V., Mavilio, F. and Sampath, K. (1994): Inhibition of proliferation and induction of differentiation of pluripotent human embryonal carcinoma cells by osteogenic protein-1 (or bone morphogenetic protein-7). Lab. Invest., 71, 243-251.

Chiang, J.Y., Kimmel, R. and Stroup, D. (2001): Regulation of cholesterol 7alpha-hydroxylase gene (CYP7A1) transcription by the liver orphan receptor (LXRalpha). Gene, 262, 257-265.

Collins, J.L., Fivush, A.M., Watson, M.A., Galardi, C.M., Lewis, M.C., Moore, L.B., Parks, D.J., Wilson, J.G., Tippin, T.K., Binz, J.G., Plunket, K.D., Morgan, D.G., Beaudet, E.J., Whitney, K.D., Kliewer, S.A. and Willson, T.M. (2002): Identification of a nonsteroidal liver $\mathrm{X}$ receptor agonist through parallel array synthesis of tertiary amines. J. Med. Chem., 45, 1963-1966.

Flaveny, C.A., Griffett, K., El-Gendy, Bel-D., Kazantzis, M., Sengupta, M., Amelio, A.L., Chatterjee, A., Walker, J., Solt, L.A., Kamenecka, T.M. and Burris, T.P. (2015): Broad anti-tumor activity of a small molecule that selectively targets the warburg effect and lipogenesis. Cancer Cell, 28, 42-56.

Fujino, T., Maruko-Ohtake, A., Ohtake, Y., Kobayashi, T., Ando, K., Takeuchi, A., Ohkubo, Y. and Hayakawa, M. (2015): Farnesoid $\mathrm{X}$ receptor knockdown provides significant growth inhibition in hepatocellular carcinoma cells while it does not interfere with the proliferation of primary human hepatocyte-derived cells. J. Toxicol. Sci., 40, 501-508.

Fujino, T., Sakamaki, R., Ito, H., Furusato, Y., Sakamoto, N., Oshima, T. and Hayakawa, M. (2017): Farnesoid X receptor regulates the growth of renal adenocarcinoma cells without affecting that of a normal renal cell-derived cell line. J. Toxicol. Sci., 42, 259-265.

Fujino, T., Takeuchi, A., Maruko-Ohtake, A., Ohtake, Y., Satoh, J., Kobayashi, T., Tanaka, T., Ito, H., Sakamaki, R., Kashimura, R., Ando, K., Nishimaki-Mogami, T., Ohkubo, Y., Kitamura, N., Sato, R., Kikugawa, K. and Hayakawa, M. (2012): Critical role of farnesoid X receptor for hepatocellular carcinoma cell proliferation. J. Biochem., 152, 577-586.

Gebhard, R.L., Clayman, R.V., Prigge, W.F., Figenshau, R., Staley, N.A., Reesey, C. and Bear, A. (1987): Abnormal cholesterol metabolism in renal clear cell carcinoma. J. Lipid Res., 28, 1177-1184

Guo, L., Song, L., Wang, Z., Zhao, W., Mao, W. and Yin, M. (2009): Panaxydol inhibits the proliferation and induces the differentiation of human hepatocarcinoma cell line HepG2. Chem. Biol. Interact., 181, 138-143.

Janowski, B.A., Willy, P.J., Devi, T.R., Falck, J.R. and Mangelsdorf, D.J. (1996): An oxysterol signalling pathway mediated by the nuclear receptor LXR alpha. Nature, 383, 728-731.

Ju, J., Wang, N., Wang, X. and Chen, F. (2015): A novel all-trans retinoic acid derivative inhibits proliferation and induces differentiation of human gastric carcinoma xenografts via up-regulating retinoic acid receptor $\beta$. Am. J. Transl. Res., 7, 856-865.

Kanai, D., Ueda, A., Akagi, T., Yokota, T. and Koide, H. (2015): Oct3/4 directly regulates expression of E2F3a in mouse embryonic stem cells. Biochem. Biophys. Res. Commun., 459, 374378.

Kennedy, M.A., Venkateswaran, A., Tarr, P.T., Xenarios, I., Kudoh, J., Shimizu, N. and Edwards, P.A. (2001): Characterization of the human ABCG1 gene: liver $\mathrm{X}$ receptor activates an internal promoter that produces a novel transcript encoding an alterna- 
Regulation of Oct3/4 expression by FXR and LXR

tive form of the protein. J. Biol. Chem., 276, 39438-39447.

Koura, M., Matsuda, T., Okuda, A., Watanabe, Y., Yamaguchi, Y., Kurobuchi, S., Matsumoto, Y. and Shibuya, K. (2015): Design, synthesis and pharmacology of 1,1-bistrifluoromethylcarbinol derivatives as liver $\mathrm{X}$ receptor $\beta$-selective agonists. Bioorg. Med. Chem. Lett., 25, 2668-2674.

Kremer-Tal, S., Narla, G., Chen, Y., Hod, E., DiFeo, A., Yea, S., Lee, J.S., Schwartz, M., Thung, S.N., Fiel, I.M., Banck, M., Zimran, E., Thorgeirsson, S.S., Mazzaferro, V., Bruix, J., Martignetti, J.A., Llovet, J.M. and Friedman, S.L. (2007): Downregulation of KLF6 is an early event in hepatocarcinogenesis, and stimulates proliferation while reducing differentiation. J. Hepatol., 46, 645-654.

Li, J., Ning, G. and Duncan, S.A. (2000): Mammalian hepatocyte differentiation requires the transcription factor HNF-4alpha. Genes Dev., 14, 464-474.

Makishima, M., Okamoto, A.Y., Repa, J.J., Tu, H., Learned, R.M., Luk, A., Hull, M.V., Lustig, K.D., Mangelsdorf, D.J. and Shan, B. (1999): Identification of a nuclear receptor for bile acids. Science, 284, 1362-1365.

Maloney, P.R., Parks, D.J., Haffner, C.D., Fivush, A.M., Chandra, G., Plunket, K.D., Creech, K.L., Moore, L.B., Wilson, J.G., Lewis, M.C., Jones, S.A. and Willson, T.M. (2000): Identification of a chemical tool for the orphan nuclear receptor FXR. J. Med. Chem., 43, 2971-2974.

Nichols, J., Zevnik, B., Anastassiadis, K., Niwa, H., Klewe-Nebenius, D., Chambers, I., Schöler, H. and Smith, A. (1998): Formation of pluripotent stem cells in the mammalian embryo depends on the POU transcription factor Oct4. Cell, 95, 379-391.

Niwa, H., Miyazaki, J. and Smith, A.G. (2000): Quantitative expression of Oct-3/4 defines differentiation, dedifferentiation or selfrenewal of ES cells. Nat. Genet., 24, 372-376.

Okamoto, K., Okazawa, H., Okuda, A., Sakai, M., Muramatsu, M. and Hamada, H. (1990): A novel octamer binding transcription factor is differentially expressed in mouse embryonic cells. Cell, 60, 461-472.

Parks, D.J., Blanchard, S.G., Bledsoe, R.K., Chandra, G., Consler, T.G., Kliewer, S.A., Stimmel, J.B., Willson, T.M., Zavacki, A.M., Moore, D.D. and Lehmann, J.M. (1999): Bile acids: natural ligands for an orphan nuclear receptor. Science, 284, 13651368.

Pawar, A., Botolin, D., Mangelsdorf, D.J. and Jump, D.B. (2003): The role of liver $\mathrm{X}$ receptor-alpha in the fatty acid regulation of hepatic gene expression. J. Biol. Chem., 278, 40736-40743.

Repa, J.J., Berge, K.E., Pomajzl, C., Richardson, J.A., Hobbs, H. and Mangelsdorf, D.J. (2002): Regulation of ATP-binding cassette sterol transporters ABCG5 and ABCG8 by the liver X receptors alpha and beta. J. Biol. Chem., 277, 18793-18800.

Rosner, M.H., Vigano, M.A., Ozato, K., Timmons, P.M., Poirier, F., Rigby, P.W. and Staudt, L.M. (1990): A POU-domain transcription factor in early stem cells and germ cells of the mammalian embryo. Nature, 345, 686-692.

Ryan, M.J., Johnson, G., Kirk, J., Fuerstenberg, S.M., Zager, R.A. and Torok-Storb, B. (1994): HK-2: an immortalized proximal tubule epithelial cell line from normal adult human kidney. Kidney Int., 45, 48-57.

Schöler, H.R., Ruppert, S., Suzuki, N., Chowdhury, K. and Gruss, P. (1990): New type of POU domain in germ line-specific protein Oct-4. Nature, 344, 435-439.

Tomizawa, M., Shinozaki, F., Motoyoshi, Y., Sugiyama, T., Yamamoto, S. and Ishige, N. (2018): Oct3/4 is potentially useful for the suppression of the proliferation and motility of hepatocellular carcinoma cells. Oncol. Lett., 16, 5243-5248.

Trieb, K., Sztankay, A., Amberger, A., Lechner, H. and GrubeckLoebenstein, B. (1994): Hyperthermia inhibits proliferation and stimulates the expression of differentiation markers in cultured thyroid carcinoma cells. Cancer Lett., 87, 65-71.

Venkateswaran, A., Laffitte, B.A., Joseph, S.B., Mak, P.A., Wilpitz, D.C., Edwards, P.A. and Tontonoz, P. (2000): Control of cellular cholesterol efflux by the nuclear oxysterol receptor LXR alpha. Proc. Natl. Acad. Sci. USA, 97, 12097-12102.

Wang, H., Chen, J., Hollister, K., Sowers, L.C. and Forman, B.M. (1999): Endogenous bile acids are ligands for the nuclear receptor FXR/BAR. Mol. Cell, 3, 543-553.

Yano, M., Hayakawa-Yano, Y. and Okano, H. (2016): RNA regulation went wrong in neurodevelopmental disorders: the example of Msi/Elavl RNA binding proteins. Int. J. Dev. Neurosci., 55, 124-130.

Zhang, Y. and Edwards, P.A. (2008): FXR signaling in metabolic disease. FEBS Lett., 582, 10-18.

Zhao, W.S., Yan, W.P., Chen, D.B., Dai L., Yang, Y.B., Kang, X.Z., Fu, H., Chen, P., Deng, K.J., Wang, X.Y., Xie, X.W., Chen, H.S. and Chen, K.N. (2019): Genome-scale CRISPR activation screening identifies a role of ELAVL2-CDKN1A axis in paclitaxel resistance in esophageal squamous cell carcinoma. Am. J. Cancer Res., 9, 1183-1200. 